\title{
Development of a real time intelligent health monitoring platform for aero-engine
}

\author{
Maria Grazia De Giorgi ${ }^{1{ }^{1 *}}$, Stefano Campilongo ${ }^{1}$, and Antonio Ficarella ${ }^{1}$ \\ ${ }^{1}$ Universy of Salento, Dept. of Engineering for Innovation, 73100, via per Monteroni LECCE, Italy.
}

\begin{abstract}
In this paper an integrated heath monitoring platform is proposed and developed for performance analysis and degradation diagnostics of gas turbine engines. In a first approach the numerical tool is able to predict engine measurable data from flight data, in order to create a dataset of expected values. Then, in the case of a mismatch between expected values and measured data coming from a real engine, a second part of the tool can be activated to detect the component under degradation. In order to evaluate the performance prediction artificial neural networks (ANN) have been implemented. The tool is able to recognize the degradation due to compressor fouling and turbine erosion. Synthetic data generation has been carried out to show how the degradation effects can affect the engine performance. The used data have been generated with a model based on gas path analysis. The training of the model is focused on components deterioration due to a combination of fouling and erosion. Different scenarios have been compared in order to carry out a sensitivity analysis and to choose the best parameters for the network input and output. Obviously the knowledge of the real engine health status can be crucial for maintenance and fleet management decisions.
\end{abstract}

\section{Introduction}

A continuous engine health monitoring coupled to an early detection of the degree of a deterioration allows more accurate fleet management ensuring both the reduction of operational and maintenance costs of an aircraft and a safer flight operation.

In this paper our aim is focused on compressor fouling and turbine blade erosion that affect significantly the overall engine performance.

There is a wide variety of fouling sources that regards all the phenomena involving hard particles smaller than $2 \mu \mathrm{m}$ : dust, dirt, sand, rust, ash and carbon particles and soft particles such as oil, unburned hydrocarbons, soot, airborne industrial chemicals, fertilizers, herbicides etc., [1].

Fouling can be classified as recoverable deterioration and can be reduced or even eliminated by cleaning through a manual cleaning, an offline or online washing [2] or through grit-blasting process.

The turbine stages are affected by erosion phenomenon, defined as material removal from the flow path components by hard abrasive particles suspended in the air or gas stream

\footnotetext{
* Corresponding author: mariagrazia.degiorgi@unisalento.it
} 
such as sand and fly ash. Erosion causes performance losses due to the decrease in surface roughness, the increase in blade tip clearance, the blunting of the blade leading edge, the thinning of the trailing edge and shortening of the blade chord [3]. In [4], [5] the authors evaluated the fan damage due to different exposure time to particles ingestion.

In the turbine section, high working temperatures make the erosion damage more severe causing an excessive thermal stress on blades surface and creep rupture. Performance degradation forecast technology for assessing degradation states quantitatively based on gas path performance parameters is one of the most important technologies, which can improve the safety, reliability, and maintenance of aero-engine [6]. A consolidated approach tracks the engine status through the monitoring of in-flight measurements such as exhaust gas temperature (EGT), spool rotational speed and overall engine vibration level (amplitude). This set of information marked the beginning of monitoring methods to insure safe operation but it is not sufficient to give the user the identification of the abnormality source [7].

The need is the generation of health indicators that cannot be directly measured; an engine model containing a degradation model can monitor, taking into account these health indicators, the engine performance. So a key factor is the relationship between health parameters and performance parameters [8].

Artificial neural networks (ANNs) are promising tools for gas turbine deterioration because are suitable for dealing with nonlinear problems and modelling complex and nonlinear dynamic systems with great flexibility and capability [9], [10].

The artificial neural network is a mathematic and logical model inspired to biological mechanism. In this study, an engine model and a performance prediction tools are proposed for a jet engine using artificial neural networks.

The engine model network takes into account engine measurable data without any information about its health status; so this ANN takes into account engine operating condition and gives as output engine measurable data. This first step can be considered an engine model able to monitor the engine performance.

The data coming from ANN engine model are compared with the data coming from real engine acquisition system, activating the diagnostics network if there is a mismatch between the two data.

The present work used for engine model calibration and validation experimental data $[11,12]$.

\section{Numerical tools for aero-engine model and degradation analysis}

\subsection{ANN for gas turbine performance and health status estimation}

Artificial Neural Networks have been used in this paper as numerical tool to build engine model and diagnostics system. A neural network consists of a number of interconnected processing nodes called neurons, which are organized in a sequence of layers, including an input layer, a single intermediate hidden layer, and an output layer.

The output layer, equal to the number of dependent variables, is calculated by weight and bias associated with connections among neurons. The intermediate layer is connected to the input and output layers.

In particular a two-layer feed-forward network with sigmoid hidden neurons and linear output neurons was implemented. The network was trained with Bayesian regularization training algorithm function. 
The optimal number of hidden neurons and of the number of epochs was obtained carrying out a sensibility analysis based on the percent error between the target value and output values obtained by using neural networks with different numbers of hidden neurons and epochs. The best performance of the neural network was achieved with 5 hidden neurons and 2000 epochs for every simulates test case.

The available flights data need to be divided into training and testing sets.

The parameter used to evaluate the ANN output estimation accuracy and indirectly to compare the different ANN input dataset is the Root Mean Square Error RMSE evaluated on the test data of each $\mathrm{j}$-th output:

$$
\left(R M S E=\sqrt{\frac{1}{n_{t s}} \sum_{i=1}^{n_{t s}}\left(t_{i}-y_{i}\right)^{2}}\right)_{j}
$$

for $j=1, \ldots . .$, no; where $t_{i}$ are the target output and $y_{i}$ the network output, $n_{t s}$ the number of data used for net test and no the number of ANN output.

In our case $\mathrm{n}_{\mathrm{ts}}=75$.

\subsection{Aero-engine model and data generation of the test cases}

In this work to test the effectiveness of the developed tool, a representative model of a turbojet is chosen, with a static thrust of $17800 \mathrm{~N}$ at sea level; all the other details are reported in $[11,12]$. The present study was carried out based on 3 samples flights denoted by Flight \#1, Flight \#2 and Flight \#3 [6]. For each flight a total of 75 sample points referred to different flight conditions were selected, 25 for each phase: climb, cruise and descend. The data of these flights have been used for the training and testing process of the machine learning tools described in the next sections.

It has been built a database of engine performance data coming from an healthy engine model. The synthetic data are generated with the combination of two software, ONX and AEDSYS [13]; the first one is used to model engine characteristics in design condition and this model has been validated on experimental data $[11,12]$, the second one is able to test the modelled engine in different mission conditions, varying altitude, Mach and thrust request.

Most of the factors that deteriorate the gas turbine performance can be simulated by changing the health parameters of the engine components. Since fouling and erosion are considered among the main cause of performance deterioration, in this study, the effects of these degradations on compressor and gas turbine will be examined.

In order to test the approach three component degradation levels have been introduced used in the validated engine model, as better described in the next section. The simulated measurements are used as the input to the prediction model to test the system's capability in assessing the changing performance and health status of the engine.

\section{Methodological Approach}

The approach of the study involves two different steps of the numerical tools. The first one runs as an engine model in order to achieve engine measurable data without any information about its health status; ANN model takes into account engine operating condition and gives as output engine measurable data. This first step can be considered an engine model able to monitor the engine performance.

Then the data coming from this model are compared with the data coming from real engine acquisition system. 
Table 1. Different data set for ANN engine performance.

\begin{tabular}{|c|c|c|c|}
\hline \multirow{2}{*}{$\begin{array}{l}\text { INPUT } \\
\text { VECTOR }\end{array}$} & \multicolumn{3}{|c|}{ PARAMETERS } \\
\hline & SET \#A1 & SET \#A2 & SET \#A3 \\
\hline Xtrain & (Alt Mach) FLIGHT 1-2 $_{2}$ & (Alt Mach \%N) Flight 1-2 & (Alt Mach Tt4)FLIGHT 1-2 \\
\hline Ytrain & (mf EGT)FLIGHT 1-2 & $(m f$ EGT)FLIGHT 1-2 & (mf EGT)FLIGHT 1-2 \\
\hline Xtest & (Alt Mach)FLIGHT 3 & (Alt Mach \%N)FLIGHT 3 & (Alt Mach Tt4)FLIGHT 3 \\
\hline Ytest & $(\mathrm{mf} \text { EGT) })_{\text {FLIGHT } 3}$ & $(\mathrm{mf} \text { EGT) })_{\text {FLIGHT } 3}$ & $(\mathrm{mf} \text { EGT) })_{\text {FLIGHT } 3}$ \\
\hline
\end{tabular}

If the two data disagree, the ANN diagnostics tool works to detect the degraded component, taking into account flight data and data from engine acquisition system and giving as output diagnostics information about components health state and helping about maintenance decisions.

\subsection{Simulation of engine performance}

As said before, the first ANN permits to calculate engine performance in various operating conditions. In this way it's possible to create a database of expected values of the engine operative parameters at different operating conditions.

Different input variables have been considered to create a neural network that could be used as an engine model. All the attempted dataset chosen as input/output are summarized in Table 1. The letter $\mathrm{X}$ and $\mathrm{Y}$ are referred to net input and output respectively.

As previously described data from Flight\#1 and Flight\#2 have been used for network training, data from Flight $\# 3$ for network testing.

The SET \#A1 involves only flight condition, namely Mach number and altitude, in the input layer of the network $[11,12]$. This dataset is not dependent from engine status but there is an evident lack about the user power demand. Rotational speed can help us to solve the problem (SET \#A2) but it can be considered a performance parameter and not a "neutral" parameter. Hence in the SET \#A3 the burner exit temperature Tt4 has been used to take into account the user request. The network outputs have been normalized respect the maximum value in order to allow the network calculation in a well-defined range $0-1$.

\subsection{ANN for aero-engine health diagnostics}

The output of the previous ANN has to be compared with the data acquired on board. If the two are not in agreement the data from the engine acquisition system can be addressed into a second network for the degradation state prediction.

The simulated scenarios (healthy and degraded components) are summarized in Table 2 where four health status scenarios can be noted. In correspondence of a healthy engine the efficiency of the compressor and the turbine are equal to their nominal values. Then three different degraded scenarios are implemented for the single component and for both the component, each with three degradation levels. In the two scenarios with the degradation of a single component, the efficiency of the other component is kept constant to its design value. 
Table 2. Engine degradation level.

\begin{tabular}{|c|c|c|c|}
\hline CONFIGURATION & $\begin{array}{c}\text { Synthetic health } \\
\text { state }\end{array}$ & Hcomp & qturb \\
\hline HEALTHY & $\overline{\mathbf{0}}$ & 0.90 & 0.89 \\
\hline \multirow{3}{*}{$\begin{array}{l}\text { COMPRESSOR } \\
\text { DEGRADATION }\end{array}$} & \multirow{3}{*}{1} & 0.85 & \multirow{3}{*}{0.89} \\
\hline & & 0.80 & \\
\hline & & 0.77 & \\
\hline \multirow{3}{*}{$\begin{array}{c}\text { TURBINE } \\
\text { DEGRADATION }\end{array}$} & \multirow{3}{*}{2} & \multirow{3}{*}{0.90} & 0.84 \\
\hline & & & 0.79 \\
\hline & & & 0.76 \\
\hline \multirow{3}{*}{$\begin{array}{c}\text { COMPRESSOR \& } \\
\text { TURBINE } \\
\text { DEGRADATION }\end{array}$} & \multirow{3}{*}{3} & 0.85 & 0.84 \\
\hline & & 0.85 & 0.79 \\
\hline & & 0.80 & 0.84 \\
\hline
\end{tabular}

\subsubsection{Engine Component Health Status Diagnostics}

We trained the neural network to identify the synthetic engine health status; in a first attempt (SET \#B1) fuel mass flow rate and exhaust gas temperature were used to identify the engine health index $[11,12]$ then in the SET \#B2 we added the Mach number to test the utility of flight condition. In the last configuration in the SET \#B3 the burner exit temperature $\mathrm{Tt} 4$, suggestive of the user request, has been introduced.

\section{Results and Discussions}

\subsection{Performance analysis results}

For the performance prediction, the neural network was built by linking flight conditions, in terms of altitude and percentage of thrust, with engine performance in terms of fuel flow rate and exhaust gas temperature.

The test set gave an independent measure of how well the network can be expected to perform on data not used to train it. The different dataset in Table 1 have been tested.

The prediction error has been measured on the data from Flight \#3, comparing fuel flow and EGT values coming from network output with the expected values of the same flight. Fig. 1 shows the trend of the two physical quantities and reports the synthetic value of the associated RMSE. Figure 1a shows that the use of altitude and Mach values is not enough to allow the network to adhere to the expected values; there is a strong divergence between the data at the beginning and in the middle of the mission. The introduction of a third parameter (SET \#A2 and SET \#A3, table 1) leads to a better fit of the target values for both (Figure $1 \mathrm{~b}$ and Figure 1c).

Table 3. Different data set for ANN engine synthetic health status diagnostics.

\begin{tabular}{|c|c|c|c|}
\hline \multirow{2}{*}{$\begin{array}{l}\text { INPUT } \\
\text { VECTOR }\end{array}$} & \multicolumn{3}{|c|}{ PARAMETERS } \\
\hline & SET \#B1 & SET \#B2 & SET \#B3 \\
\hline $\mathbf{X}_{\text {train }}$ & $(\mathrm{mf}$ EGT) FLIGHT 1-2 & (Mach mf EGT)FLIGHT 1-2 & (Mach mf EGT Tt4)FLIGHT 1-2 \\
\hline$Y_{\text {train }}$ & $\left(\begin{array}{llll}0 & 1 & 2 & 3\end{array}\right)_{\text {FLIGHT 1-2 }}$ & $\left(\begin{array}{llll}0 & 1 & 2 & 3\end{array}\right)_{\text {FLIGHT 1-2 }}$ & 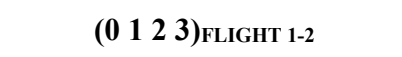 \\
\hline $\mathbf{X}_{\text {test }}$ & (mf EGT)FLIGHT 3 & (Mach mf EGT)FLIGHT 3 & (Mach mf EGT Tt4)FLIGHT 3 \\
\hline $\mathbf{Y}_{\text {test }}$ & $\left(\begin{array}{llll}0 & 1 & 2 & 3\end{array}\right)_{\text {FLIGHT }} 3$ & $\left(\begin{array}{llll}0 & 1 & 2 & 3\end{array}\right)_{\text {FLIGHT }} 3$ & $\left(\begin{array}{llll}0 & 1 & 2 & 3\end{array}\right)_{\text {FLIGHT }} 3$ \\
\hline
\end{tabular}


It's evident that the use of a physical quantity related to the usage demand is a key factor for a good response from the neural network; in particular the burner exit temperature, used in the SET \#A3 (Fig. 1c), is related to the leverage position, usually a quantity not acquired in the practice.

It's notable that the network needs only three input parameters to give very good results.

\subsection{Results of Engine Component Health Status Prediction}

In this section the synthetic health state for the engine has been estimated to evaluate the engine component's status. Based on Table 2 that reports the health state conditions, we can assert if the engine is healthy (0), or if there is a degradation of the compressor (1), of the turbine (2) or of both these two components (3).
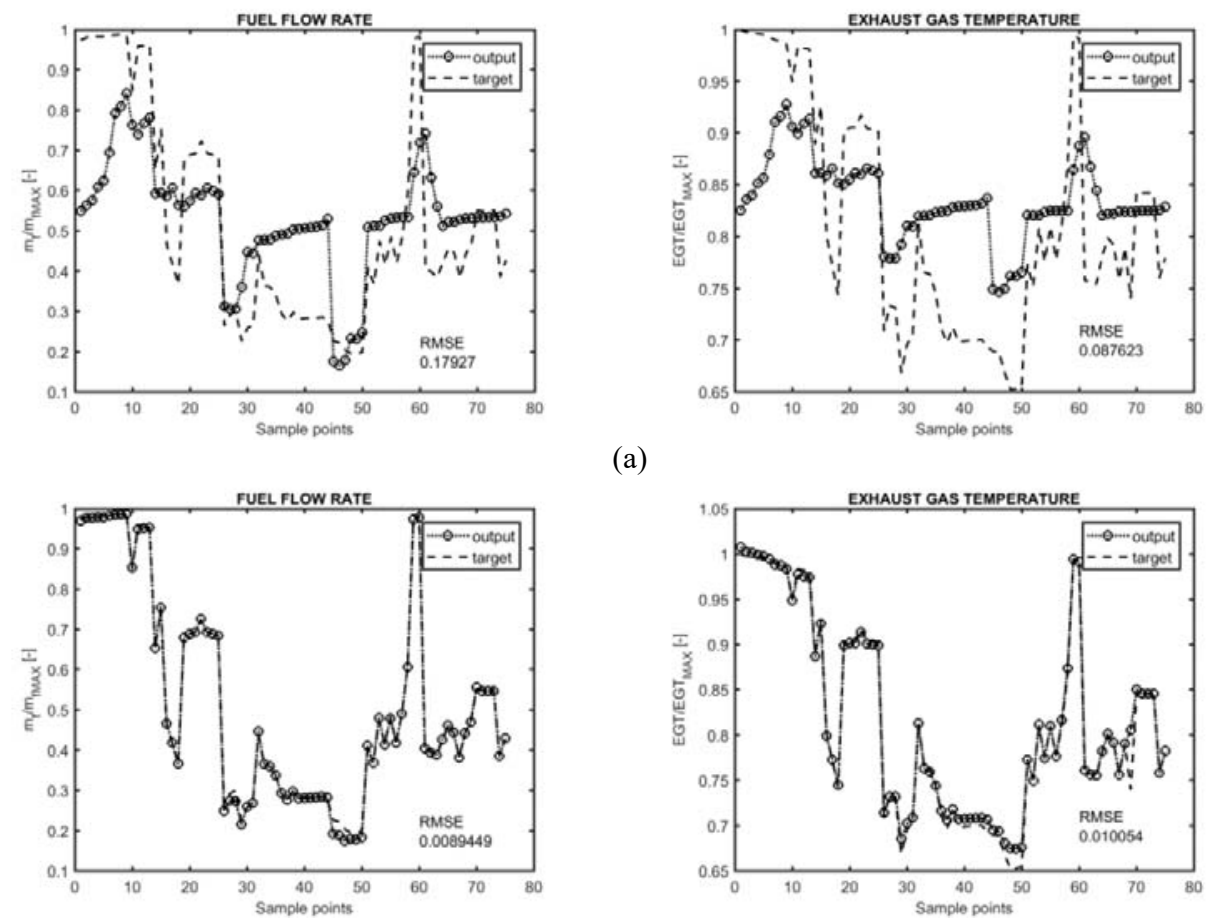

(a)

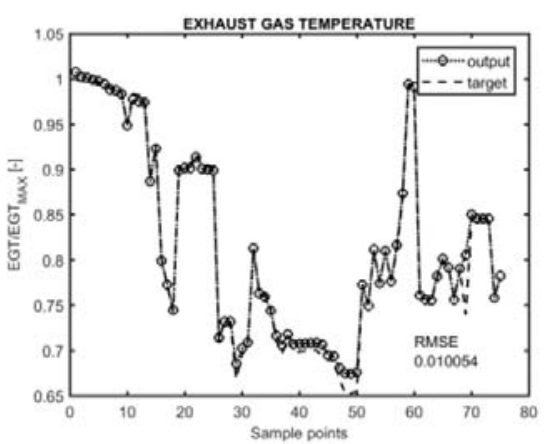

(b)
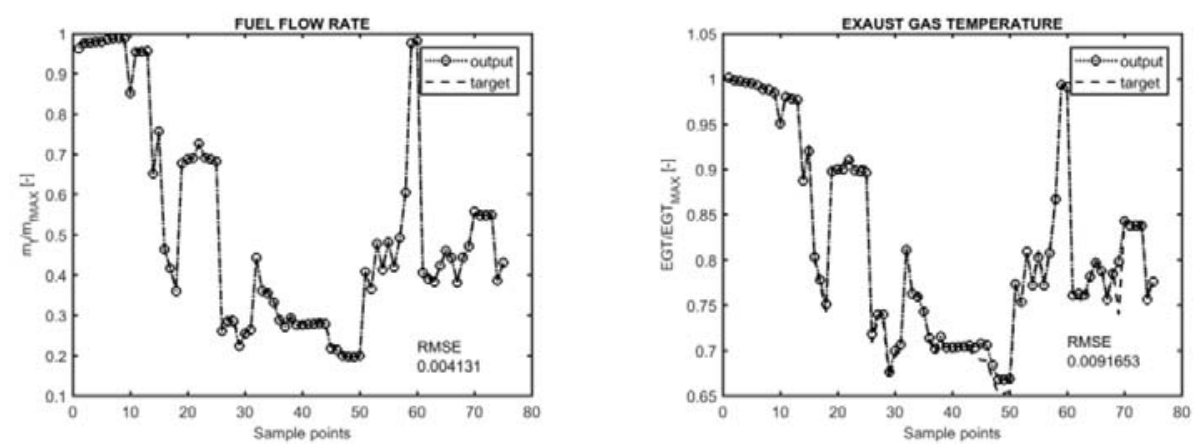

(c)

Fig. 1. Performance prediction results for SET \#A1 (a), SET \#A2 (b) and SET \#A3 (c). 


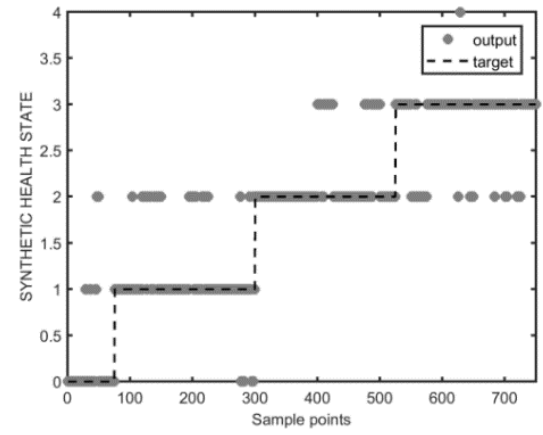

(a)

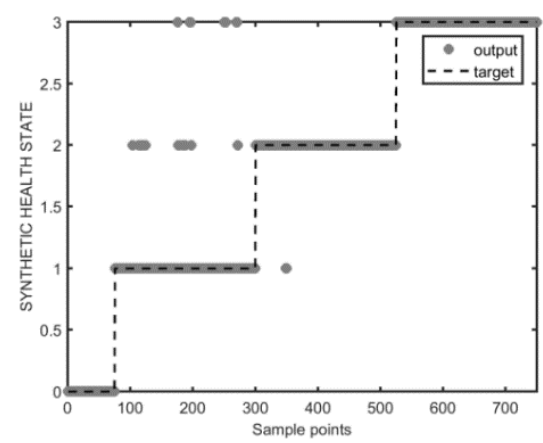

(c)

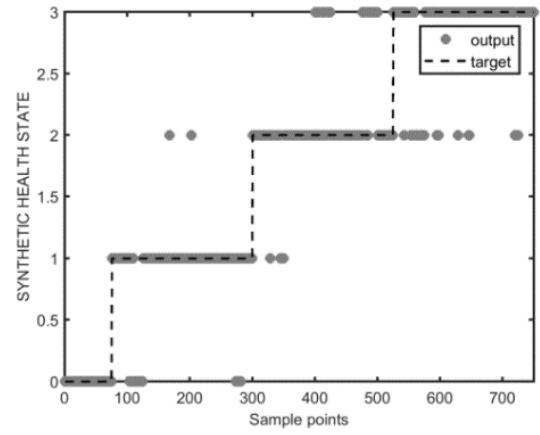

(b)

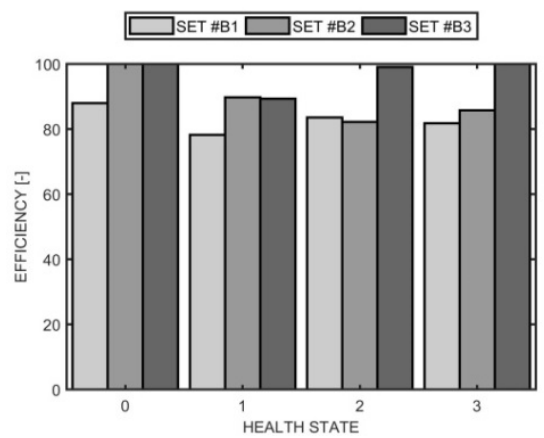

(d)

Fig. 2. Engine synthetic health status prediction for SET \#B1 (a), SET \#B2 (b) and SET \#B3 (c); Efficiency of health state prediction (d)

The performance of the different datasets, which are listed in Table 3, have been tested by using some engine data parameters as inputs and the previously described synthetic health state as output. Fig. 2a-2b-2c show the trend of network output with respect to expected values; the efficiency of the prediction tool is depicted with histograms in the Figure $2 \mathrm{~d}$; in this case the network efficiency is calculated counting the number of well predicted health state values with respects to the total values. In the efficiency graph (Figure 2d) the performance of the prediction tools has been evaluated for the different synthetic health state conditions. Obviously, dividing the results in zones with different degraded scenario we are able to detect the degraded component even if it's impossible to quantify its deterioration level giving us only an alert about the engine health condition.

In Fig. 2a the results for the SET \#B1are nearly constant for all the scenarios with a mean efficiency value of $82 \%$. The SET \#B2 (Fig. 2b) shows that with the addition of the Mach number in the input vector, the prediction of a healthy engine is very good $(99.6 \%)$, but there is still a lack for the output degraded conditions 1, 2 and 3. In detail the synthetic health state 2 is misclassified with the synthetic health state 3 and vice versa. The mean efficiency value of the SET \#B2 is equal to $87,3 \%$.

The best results have been performed by the SET \#B3 (Fig. 2c) with a mean efficiency value of $96,5 \%$; this dataset presented only few wrong points for the health state 1 , misclassified mainly with the health state 2 and for few points with the health state 3 . The SET\#B3 is a compromise between performance parameters, such as fuel flow rate and exhaust gas temperature, and flight parameters, such as Mach number and burner exit temperature. 


\section{Conclusions}

This work is a first step to create a tool for monitoring aero-engine health status. The long-term objective of the research will be a data-driven maintenance, which can be carried out with an innovative methodological analysis of the big data cluster from aero-engine system. The ANN based tool that works as engine model has shown very good results in terms of engine performance parameters calculated from only few operational data. The very small computational time for this tool allows its use of this net in real-time condition.

The tool is able to recognize the degradation of the two main gas turbine engine components: compressor and turbine. Different scenarios have been compared in order to carry out a sensitivity analysis and to choose the best parameters for the network input and output. The best results have been performed by the input vector based on (Mach, fuel flow rate $\mathrm{mf}$, EGT and inlet turbine temperature) with a mean prediction efficiency value of $96,5 \%$ involving in the input vector fuel flow rate, exhaust gas temperature, Mach number and burner exit temperature.

\section{References}

1. R. Kurz, K. Brun, J. Eng. Gas Turb. Power, 123, 70-77 (2001)

2. C.B. Meher-Homji, M. Chaker, A. F. Bromley, Proceedings of ASME Turbo Expo: Power for Land, Sea and Air, 4, 571-590 (2009)

3. A. A. Hamed, W. Tabakoff, R. B. Rivir, K. Das, P. Arora, J. Turbomach., 127, 445452, (2005)

4. M. G. De Giorgi, S. Campilongo, A. Ficarella, M. Coltelli, V. Pfister, F. Sepe, Proceedings of the ASME Turbo Expo, GT2013-95662, (2013)

5. M. G. De Giorgi, S. Campilongo, A. Ficarella, A., J. Eng. Gas Turb. Power, 137, 052603-1-15, (2014)

6. M. G. De Giorgi, L. De Carlo, A. Ficarella, 7th EASN International Conference (2017)

7. A. J. Volponi, J. Eng. Gas Turb. Power, 136, (051201) 1-20, (2014)

8. G. P. Sallee, Performance Deterioration Based on Existing (Historical) Data - JT9D Jet Engine Diagnostics Program, NASA CR-135448, (1978)

9. S. S. Tayarani-Bathaie, Z. N. Sadough Vanini, K. Khorasani, Conference Proceedings in Canadian Conference on Electrical and Computer Engineering, (2012)

10. O. E. Dragomir, R. Gouriveau, F. Dragomir, E. Minca, N. Zerhouni, European control conference (ECC) IEEE: 1587-92, (2009)

11. M. G. De Giorgi, L. De Carlo, A. Ficarella A., Aircr. Eng. Aerosp. Tec., (2018)

12. M. G. De Giorgi, S. Campilongo, A. Ficarella, Enrgy Proced, 10.1016/j.egypro.2018.08.109.

13. J. D. Mattingly, W. H. Heiser, D. T. Pratt, Aircraft Engine Design, Second Edition by the American Institute of Aeronautics and Astronautics, Inc, (2002) 